\title{
Efficient Implementation of Non-linear Valve Law and Ventricular Interaction Dynamics in the Minimal Cardiac Model
}

\author{
C. E. Hann ${ }^{1}$, J. G. Chase ${ }^{2}$, G. M. Shaw ${ }^{3}$ \\ Department of Mechanical Engineering \\ University of Canterbury \\ Private Bag 4800 \\ Christchurch \\ New Zealand
}

Email: Chris.Hann@canterbury.ac.nz

\footnotetext{
${ }^{1}$ Research Associate, New Zealand Science and Technology Postdoctoral Fellow, Dept. of Mech. Eng

${ }^{2}$ Assoc. Prof./Reader, Dept of Mech. Eng

${ }^{3}$ Consultant, Christchurch Hospital Dept of Intensive Care Medicine, Christchurch, New Zealand
} 


\begin{abstract}
A minimal model of the cardio-vascular system (CVS) with ventricular interaction and inertial effects that accurately captures the physiological trends of a variety of disease states has been developed. However, the physiologically accurate open on pressure, close on flow valve law is computationally heavy to implement, reducing the model's potential clinical benefit. A significantly simpler representation of the valve law using Heaviside functions is derived and the ventricular interaction equations are reformulated to obtain a unique closed form analytical solution. The new formulation is tested and compared with the previous formulation for a healthy human and four clinically significant disease states: mitral and aortic stenosis, pulmonary embolism and septic shock. The new model formulation matches the previous model definition, differing by a mean model response error of no more than $0.2 \%$. Computationally, it is $24 \times$ faster than the previous method. More specifically, a short 20-beat simulation that took 102 seconds now requires 4.3 seconds, significantly improving the model's potential for practical use in a diagnostic and/or decision support role in the intensive care unit.
\end{abstract}

\title{
1 Introduction
}

Heart disease is difficult to accurately diagnose and treat in an intensive care unit (ICU). Medical staff have to analyze a wide range of, sometimes conflicting, data on a patient's condition, which often never directly reveals the problem or best treatment to apply. The body's natural reflex actions to restore equilibrium can often mask the actual problem, giving rise to many combinations that medical staff have to consider. Hence, the ICU clinical necessity of making a quick diagnosis leads to decisions that rely primarily on the experience and intuition of clinical staff.

Modelling is a well known method of assisting in understanding the mechanisms involved in cardio-vascular system (CVS) dysfunction. Most current approaches to 
modelling the CVS can be grouped into either Finite Element (FE) or PressureVolume (PV) approaches. FE techniques offer potentially very accurate results but require very detailed patient specific information such as muscle fibre orientations, structures and mechanical properties which is not readily available in intensive care [1-4]. Furthermore, the computational power required is currently too extreme for clinical use. PV methods divide the CVS system into a series of elastic chambers separated by resistances, and inertial effects where required. Each elastic chamber models a section of the CVS such as the ventricles, the atria, or the aorta, each with their own pressure-volume relationship. Only a minimal number of parameters, such as chamber elastances and arterial resistances, are required. These models are more suitable for clinical feedback but most are focused on simulating only certain areas of CVS function [5-10].

A minimal model has been developed which accurately simulates a variety of CVS dysfunctions and has been shown to be both stable and accurate [11-13]. The idea is to first simulate a specific patient's condition by finding the patient specific parameters that give the best model match to measured pressures, volumes and flows of that patient [14]. The resulting patient specific model can then be used to test a variety of therapy treatments to assist medical staff in finding the optimum treatment. To be of practical clinical use though, this process must be done in a clinically useful time (3-5 minutes maximum).

An integral based patient specific parameter identification method for physiological system models has been developed $[14,15]$ that enables staff to rapidly obtain the required patient specific model. However, there still remains the task of trialling many different therapies, which could require potentially many simulations of the model. Thus, it is critically important to have a very efficient, easily implementable method for simulating the full heart model that can be performed on a standard desktop computer. This requirement increases if the model is to have the computational head room to evolve as more clinical data becomes available over time. 
This paper reformulates the full model in terms of Heaviside functions which significantly simplifies the implementation and the ventricular interaction equation is reformulated giving a closed form analytical solution. This new formulation results in significantly large computational savings over the previous formulation [11-13].

\section{Methodology}

\subsection{Cardiac Chamber Models:}

The differential equations describing the flows $Q_{1}$ and $Q_{2}$ in and out of the single elastic chamber shown in Figure 1, with inertia and upstream and downstream pressures, $P_{1}$ and $P_{3}$, are defined [12]:

$$
\begin{aligned}
\dot{V} & =Q_{1}-Q_{2} \\
\dot{Q}_{1} & =\frac{P_{1}-P_{2}-Q_{1} R_{1}}{L_{1}} \\
\dot{Q}_{2} & =\frac{P_{2}-P_{3}-Q_{2} R_{2}}{L_{2}}
\end{aligned}
$$

where $Q_{1}$ and $Q_{2}$ are the flows in and out, $L_{1}$ and $L_{2}$ are inertances of the blood, and $R_{1}$ and $R_{2}$ are resistances. The driving pressure in the chamber is defined:

$$
\begin{aligned}
P_{2} & =e(t) E_{\mathrm{es}}\left(V-V_{d}\right)+(1-e(t)) P_{0}\left(e^{\lambda\left(V-V_{0}\right)}-1\right), \\
e(t) & =e^{-80(t-0.375)^{2}}
\end{aligned}
$$

where $E_{\text {es }}$ is elastance, $V_{d}$ is volume at zero pressure, $e(t)$ is a driving function that simulates ventricular contraction, and $P_{0}, \lambda$, and $V_{0}$ define gradient, curvature and volume at zero pressure of the EDPVR curve in the cardiac cycle shown in Figure $2[12]$.

Equations (1) and (2) are solved when $Q_{1}>0$, during the filling stage, and Equations (1) and (3) are solved when $Q_{2}>0$, during the ejection stage. In between 
these states the model is much simpler during the iso-volumetric expansion and contraction phase. During these phases volume and pressure are simply related by Equations (4) and (5). Finally, this model employs an open on pressure, close on flow valve law as shown in Figure $2[11,13]$, which also shows the model states used for simulating each portion of the cardiac cycle.

\subsubsection{Full model definition:}

With active chambers and elements as in Figure 1 and added passive chambers, a full CVS model can be readily assembled, as shown in Figure 3. For the full model, the volume in the left and right ventricles are described by the differential equations:

$$
\begin{gathered}
\dot{V}_{\mathrm{lv}}=Q_{\mathrm{mt}}-Q_{\mathrm{av}} \\
\dot{V}_{\mathrm{rv}}=Q_{\mathrm{tc}}-Q_{\mathrm{pv}} .
\end{gathered}
$$

The differential equations describing the flows through the aortic valve and mitral valve on either side of the left ventricle can be written for the full model [11-13]:

$$
\begin{gathered}
L_{\mathrm{av}} \dot{Q}_{\mathrm{av}}=P_{\mathrm{lv}}-P_{\mathrm{ao}}-R_{\mathrm{av}} Q_{\mathrm{av}} \\
L_{\mathrm{mt}} \dot{Q}_{\mathrm{mt}}=P_{\mathrm{pu}}-P_{\mathrm{lv}}-R_{\mathrm{mt}} Q_{\mathrm{mt}}
\end{gathered}
$$


The remaining pressure, volume and flow relationships are defined [11-13]:

$$
\begin{aligned}
P_{\mathrm{lv}} & =P_{\mathrm{lvf}}+P_{\mathrm{peri}} \\
P_{\mathrm{lvf}} & =e(t) E_{\mathrm{es}, \mathrm{lvf}} V_{\mathrm{lvf}}+(1-e(t)) P_{0, \mathrm{lvf}}\left(e^{\lambda_{\mathrm{lvf}} V_{\mathrm{lvf}}}-1\right) \\
V_{\mathrm{lvf}} & =V_{\mathrm{lv}}-V_{\mathrm{spt}} \\
P_{\mathrm{peri}} & =P_{\mathrm{pcd}}+P_{\mathrm{th}} \\
P_{\mathrm{pcd}} & =P_{0, \mathrm{pcd}}\left(e^{\lambda_{\mathrm{pcd}}\left(V_{\mathrm{pcd}}-V_{0, \mathrm{pcd}}\right)}-1\right) \\
V_{\mathrm{pcd}} & =V_{\mathrm{lv}}+V_{\mathrm{rv}} \\
P_{\mathrm{ao}} & =E_{\mathrm{ao}} V_{\mathrm{ao}} \\
P_{\mathrm{pu}} & =E_{\mathrm{pu}} V_{\mathrm{pu}}+P_{\mathrm{th}} \\
\dot{V}_{\mathrm{ao}} & =Q_{\mathrm{av}}-Q_{\mathrm{sys}} \\
\dot{V}_{\mathrm{pu}} & =Q_{\mathrm{pul}}-Q_{\mathrm{mt}}
\end{aligned}
$$

where $E_{j}$ denotes the $j^{\text {th }}$ chamber elastance, and all the other variables are defined in Figure 3. Finally, the circulatory flow rates $Q_{\text {sys }}$ and $Q_{\text {pul }}$ can be defined:

$$
\begin{aligned}
Q_{\mathrm{sys}} & =\frac{P_{\mathrm{ao}}-P_{\mathrm{vc}}}{R_{\mathrm{sys}}} \\
Q_{\mathrm{pul}} & =\frac{P_{\mathrm{pa}}-P_{\mathrm{pu}}}{R_{\mathrm{pul}}}
\end{aligned}
$$

and the other variables are defined in Figure 3.

To finish the model definition, the pressures in the pulmonary artery $\left(P_{\mathrm{pu}}\right)$ and vena cava $\left(P_{\mathrm{vc}}\right)$ passive chambers in Figure 3 are defined:

$$
\begin{aligned}
& P_{\mathrm{vc}}=E_{\mathrm{vc}} V_{\mathrm{vc}} \\
& P_{\mathrm{pa}}=E_{\mathrm{pa}} V_{\mathrm{pa}}+P_{\mathrm{th}}
\end{aligned}
$$

The method described in $[11,12]$, uses an event solver to detect when a valve 
should be opened or closed, and then it breaks out of the simulation to use a new model as defined in Figure 2. For the full model described by Equations (6)-(23) there are two valves for each of the left and right ventricles giving rise to a number of combinations of open and closed positions of the valves to capture. This formulation can be coded, but takes some effort where the event solver is computationally heavy, constantly searching for sign changes in model states. Another significantly simpler formulation that does not require an event solver is to automatically account for the valve opening or closing using Heaviside functions.

\subsection{Heaviside model formulation}

For simplicity the Heaviside formulation is explained using the single chamber Equations (1)-(5) for the case of the left ventricle and constant upstream and downstream pressures. The same approach can be readily extended to the full model of Equations (6)-(23).

\subsubsection{Left ventricle}

For the left ventricle the upstream pressure $P_{1}$ is the pressure in the pulmonary vein $\left(P_{\mathrm{pu}}\right)$ and the downstream pressure $P_{2}$ is the pressure in the aorta $\left(P_{\mathrm{ao}}\right)$. As an approximation $P_{1}$ is set to $2 \mathrm{mmHg}$ and $P_{3}$ is set to $100 \mathrm{mmHg}$ representing the average pressures in the pulmonary vein and aorta for a healthy human [16]. The pressure in the left ventricle $P_{2}$ is assumed to be initially a small value with $P_{2}<P_{1}$. Consider the following Heaviside formulation of Equations (1)-(3):

$$
\begin{aligned}
\dot{V} & =H\left(Q_{1}\right) Q_{1}-H\left(Q_{2}\right) Q_{2} \\
\dot{Q}_{1} & =H\left(H\left(P_{1}-P_{2}\right)+H\left(Q_{1}\right)-0.5\right) \frac{\left(P_{1}-P_{2}-R_{1} Q_{1}\right)}{L_{1}} \\
\dot{Q}_{2} & =H\left(H\left(P_{2}-P_{3}\right)+H\left(Q_{2}\right)-0.5\right) \frac{\left(P_{2}-P_{3}-R_{2} Q_{2}\right)}{L_{2}}
\end{aligned}
$$


where the Heaviside function $H(K(t))$ as used, is defined:

$$
\begin{array}{rlrl}
H(K(t)) & =0, & K(t) \leq 0 \\
& =1, \quad K(t)>0
\end{array}
$$

Initially, if $Q_{1}(0)=0, Q_{2}(0)=0, P_{2}<P_{1}, P_{2}<P_{3}$ is assumed, the results yield:

$$
\begin{aligned}
& \dot{Q}_{1}=H(1+0-0.5) \frac{\left(P_{1}-P_{2}-R_{2} Q_{2}\right)}{L_{1}}=\frac{P_{1}-P_{2}}{L_{1}}>0 \\
& \dot{Q}_{2}=H(0+0-0.5) \frac{\left(P_{2}-P_{3}-R_{2} Q_{2}\right)}{L_{2}}=0
\end{aligned}
$$

Note that the -0.5 term in Equations (25) and (26) is employed so that if a Heaviside function is used that is undefined at 0 as in MATLAB7.01 for example, then an error will not be produced when $H\left(P_{2}-P_{3}\right)+H\left(Q_{2}\right)=0$ in Equation (26).

The solution to $\dot{Q}_{2}=0$ with an initial condition of $Q_{2}(0)=0$ is $Q_{2}=0$. Thus when Equations (24)-(26) are numerically solved simultaneously, $Q_{2}$ remains at 0 while $Q_{1}$ becomes positive because $\dot{Q}_{1}>0$ as stated in Equation (29). By Equation (24), $\dot{V}>0$ and $V$ will increase. This result corresponds to the filling phase of the left ventricle. The pressure $P_{2}$ will then increase as described in Figure 2, but when $P_{2}$ becomes greater than $P_{1}$, the inlet valve does not shut off (that is $\dot{Q}_{1}=0$ ) until $Q_{1}$ becomes 0 or negative. This implementation of the close on flow portion of the valve law occurs because:

$$
\begin{aligned}
H\left(H\left(P_{1}-P_{2}\right)+H\left(Q_{1}\right)-0.5\right) & =1, \quad P_{2}>P_{1} \text { and } Q_{1}>0 \\
& =0, \quad P_{2}>P_{1} \text { and } Q_{1} \leq 0
\end{aligned}
$$

Hence, this valve law captures the effect of inertia for the inlet valve by closing on flow.

At the point where Equation (32) first holds, $\dot{Q}_{1}=0$ and depending on the stepsize of the DE solver, $Q_{1}$ is a small negative number, typically of the order of 
$-1 \times 10^{-4}$ to $-1 \times 10^{-6}$.

The DE solver then proceeds into the iso-volumetric contraction phase, and two differential equations exist from the point $t=t_{1}$, where $t_{1}$ is the time step where $Q_{1}$ first goes negative.

$$
\begin{aligned}
& \dot{Q}_{1}=0, \quad Q_{1}\left(t_{1}\right)=-1 \times 10^{-4} \text { (for example) } \\
& \dot{Q}_{2}=0, \quad Q_{2}\left(t_{1}\right)=0
\end{aligned}
$$

Hence, $Q_{1}$ and $Q_{2}$ will be held at $-1 \times 10^{-4}$ and 0 , and $\dot{V}=0$ by Equation (24). During contraction, $V$ stays constant and the pressure $P_{2}$ continues to increase until $P_{2}$ becomes greater than $P_{3}$ at the beginning of the ejection phase. In this phase, $\dot{Q}_{1}$ remains at 0, thus opening on pressure since Equation (32) still holds and $Q_{2}$ is 0 , but is now defined:

$$
\dot{Q}_{2}=H(1+0-0.5) \frac{\left(P_{2}-P_{3}-R_{2} Q_{2}\right)}{L_{2}}=\frac{P_{2}-P_{3}}{L_{2}}>0
$$

Thus, as the DE solver continues, $Q_{2}$ increases above 0. By Equation (24), since $Q_{1}=-1 \times 10^{-4}$ and $Q_{2}>0, \dot{V}<0$ and $V$ will increase. The pressure $P_{2}$ will then decrease as described in Figure 2, but in a similar way to the inlet valve, when $P_{2}$ becomes smaller than $P_{3}$ the outlet valve does not shut off (that is $\dot{Q}_{2}=0$ ) until $Q_{2}$ itself becomes 0 or negative and closes on flow. This result occurs because:

$$
\begin{aligned}
H\left(H\left(P_{2}-P_{3}\right)+H\left(Q_{2}\right)-0.5\right) & =1, \quad P_{2}<P_{3} \text { and } Q_{2}>0 \\
& =0, \quad P_{2}<P_{3} \text { and } Q_{2} \leq 0
\end{aligned}
$$

At the point where Equation (37) first holds, $\dot{Q}_{2}=0$ and depending on the stepsize of the DE solver, $Q_{2}$ is a small negative number, typically of the order of $-1 \times 10^{-4}$ to $-1 \times 10^{-6}$. The DE solver then proceeds into isovolumetric expansion with the two differential equations from the point $t=t_{2}$, where $t_{2}$ is the time step 
where $Q_{2}$ first goes negative:

$$
\begin{array}{ll}
\dot{Q}_{1}=0, & Q_{1}\left(t_{2}\right)=-1 \times 10^{-4} \text { (for example) } \\
\dot{Q}_{2}=0, & Q_{2}\left(t_{2}\right)=-1 \times 10^{-4} \text { (for example) }
\end{array}
$$

Hence, $Q_{1}$ and $Q_{2}$ will be both held at $-1 \times 10^{-4}$ and $\dot{V}=0$ by Equation (24), $V$ stays constant, and the pressure $P_{2}$ continues to decrease until $P_{2}<P_{1}$, which starts the whole cardiac cycle process again.

This cycle is continued for as many heart beats as required. Thus, the two flow differential equations and the volume differential equation are solved simultaneously for all time without needing the event solver to switch models and sets of equations, as shown in Figure 2 for the work in [11-13]. All that is required are initial conditions at the start.

By avoiding switching models and equations the small errors that occur with an event solver will not build up over long simulations and contaminate the results and model stability.

\subsubsection{Full model Heaviside formulation}

For the full model the valve law in each of the mitral, aortic, pulmonary and tricuspid valves can be represented using Heavisides in a similar way as described for Equations (24)-(26). The differential equations for the left ventricle given by Equations (8), (9), (18) and (19) become:

$$
\begin{aligned}
& \dot{Q}_{\mathrm{av}}=H\left(H\left(P_{\mathrm{lv}}-P_{\mathrm{ao}}\right)+H\left(Q_{\mathrm{av}}\right)-0.5\right) \frac{\left(P_{\mathrm{lv}}-P_{\mathrm{ao}}-R_{\mathrm{av}} Q_{\mathrm{av}}\right)}{L_{\mathrm{av}}} \\
& \dot{Q}_{\mathrm{mt}}=H\left(H\left(P_{\mathrm{pu}}-P_{\mathrm{lv}}\right)+H\left(Q_{\mathrm{mt}}\right)-0.5\right) \frac{\left(P_{\mathrm{pu}}-P_{\mathrm{lv}}-R_{\mathrm{mt}} Q_{\mathrm{mt}}\right)}{L_{\mathrm{mt}}} \\
& \dot{V}_{\mathrm{ao}}=H\left(Q_{\mathrm{av}}\right) Q_{\mathrm{av}}-Q_{\mathrm{sys}} \\
& \dot{V}_{\mathrm{pu}}=Q_{\mathrm{pul}}-H\left(Q_{\mathrm{mt}}\right) Q_{\mathrm{mt}}
\end{aligned}
$$


Equations (40)-(43) are then combined with Equations (10)-(17), which are unchanged. The Heaviside formulation of the differential equations for the right ventricle can be written in a similar way.

\subsection{Simpler Heaviside formulation}

Equations (24)-(26) contain embedded Heavisides that can slow the DE solver down, adding computational cost. In this section a new formulation is presented that is essentially equivalent to Equations (24)-(26) in the filling and ejection stages, but does not require the embedded Heaviside functions.

Consider the following set of differential equations for a single chamber defined:

$$
\begin{aligned}
\dot{V} & =H\left(Q_{1}\right) Q_{1}-H\left(Q_{2}\right) Q_{2} \\
\dot{Q}_{1} & =\frac{P_{1}-P_{2}-R_{1} Q_{1}}{L_{1}} \\
\dot{Q}_{2} & =\frac{P_{2}-P_{3}-R_{2} Q_{2}}{L_{2}}
\end{aligned}
$$

where in comparison to Equations (40)-(43) fewer Heaviside functions are used.

For the case of the left ventricle, with constant upstream and downstream pressures as looked at in section 2.2.1, the initial conditions are $Q_{1}=0, Q_{2}=0$ and $P_{2}<P_{1}<P_{3}$. As Equations (44)-(46) are solved, $\dot{Q}_{2}<0$ so $Q_{2}$ decreases to become negative such that:

$$
\dot{V}=H\left(Q_{1}\right) Q_{1}-H\left(Q_{2}\right) Q_{2}=1 \cdot Q_{1}-0 \cdot Q_{2}=Q_{1}
$$

Since $\dot{Q}_{1}>0, Q_{1}$ and $V$ increase, which is the filling stage, and thus at this point both Equations (44)-(46) and Equations (24)-(26) give the same solution for $V$ and $Q_{1}$. Eventually, $Q_{1}$ hits zero and becomes negative, so that by Equation (44), $\dot{V}=0 \cdot Q_{1}-0 \cdot Q_{2}=0$. As $P_{2}$ increases $P_{2}-P_{3}$ becomes less negative and since $Q_{2}$ 
is negative, $\left|P_{2}-P_{3}\right|$ will become sufficiently small such that

$$
\dot{Q}_{2}=\frac{P_{2}-P_{3}-R_{2} Q_{2}}{L_{2}}>0
$$

Thus, $Q_{2}$ will increase and become less negative. But when $P_{2}=P_{3}, Q_{2}$ is not necessarily at 0 as it would be if Equations (24)-(26) were solved. When $P_{2}$ first becomes greater than $P_{1}$, Equation (26) is the same as Equation (46), which has the analytical solution:

$$
Q_{2}(t)=Q_{2}\left(t_{1}\right) e^{-\frac{R_{2}}{L_{2}}\left(t-t_{1}\right)}+\frac{1}{L_{2}} \int_{t_{1}}^{t} e^{-\frac{R_{2}}{L_{2}}(t-\tau)}\left(P_{2}(\tau)-P_{3}\right) d \tau
$$

where $t_{1}$ is the time where $P_{2}$ first equals $P_{3}$.

The difference between the solutions for $Q_{2}$ is that for Equations (24)-(26), $Q_{2}\left(t_{1}\right)=0$ where for Equations (44)-(46), $Q_{2}\left(t_{1}\right) \neq 0$. Since $L_{2}<<R_{2}$ (inductances are approximately a factor of 100 smaller than resistances of the valves [11]) there is a very small time constant of the order of $0.01 \mathrm{~s}$ so that the two solutions will converge quickly. This portion corresponds to the ejection stage. Eventually $Q_{2}$ will hit zero and become negative closing the valve.

A similar analytical construction can be done for $Q_{1}$ to show that $Q_{1}$ converges quickly onto the solution of Equations (24)-(26) during the filling stage. This process continues for as many heart beats as required. Note that in practice, this simpler Heaviside formulation could be run for a number of heart beats until the solution settles to a steady state and then Equations (24)-(26) could be simulated for one more heart beat to correct for the error in this transient period at the beginning stages of filling and ejection. 
For the full model the equations for the left ventricle in this approach are defined:

$$
\begin{aligned}
& \dot{Q}_{\mathrm{av}}=\frac{\left(P_{\mathrm{lv}}-P_{\mathrm{ao}}-R_{\mathrm{av}} Q_{\mathrm{av}}\right)}{L_{\mathrm{av}}} \\
& \dot{Q}_{\mathrm{mt}}=\frac{\left(P_{\mathrm{pu}}-P_{\mathrm{lv}}-R_{\mathrm{mt}} Q_{\mathrm{mt}}\right)}{L_{\mathrm{mt}}} \\
& \dot{V}_{\mathrm{ao}}=H\left(Q_{\mathrm{av}}\right) Q_{\mathrm{av}}-Q_{\mathrm{sys}} \\
& \dot{V}_{\mathrm{pu}}=Q_{\mathrm{pul}}-H\left(Q_{\mathrm{mt}}\right) Q_{\mathrm{mt}}
\end{aligned}
$$

The equations for the right ventricle can be written in a similar way with similar results and the other Equations for the full system are unchanged.

\subsection{Ventricular Interaction}

Ventricular interaction is an importnat dynamic in obtaining accurate CVS dynamics $[11,13]$. The septum volume, $V_{\mathrm{spt}}$, is calculated from numerically solving the equation $[11,12]$ :

$$
\begin{aligned}
& e(t) E_{\mathrm{es}, \mathrm{spt}}\left(V_{\mathrm{spt}}-V_{\mathrm{d}, \mathrm{spt}}\right)+(1-e(t)) P_{0, \mathrm{spt}}\left(e^{\lambda_{\mathrm{spt}}\left(V_{\mathrm{spt}}-V_{0, \mathrm{spt}}\right)}-1\right) \\
& =e(t) E_{\mathrm{es}, \mathrm{lvf}}\left(V_{\mathrm{lv}}-V_{\mathrm{spt}}\right)+(1-e(t)) P_{0, \mathrm{lvf}}\left(e^{\lambda_{\mathrm{lvf}}\left(V_{\mathrm{lv}}-V_{\mathrm{spt}}\right)}-1\right) \\
& -e(t) E_{\mathrm{es}, \mathrm{rvf}}\left(V_{\mathrm{rv}}+V_{\mathrm{spt}}\right)-(1-e(t)) P_{0, \mathrm{rvf}}\left(e^{\lambda_{\mathrm{rvf}}\left(V_{\mathrm{rv}}+V_{\mathrm{spt}}\right)}-1\right)
\end{aligned}
$$

at each time step in the numerical differential equation routine, where $E_{\text {es,spt }}$, $P_{0, \text { spt }}, \lambda_{\mathrm{spt}}, V_{\mathrm{d}, \mathrm{spt}}, V_{0, \mathrm{spt}}$ are fixed generic parameters [11-13].

Due to the high non-linearities in Equation (54) this procedure is very computationally expensive. As Equation (54) stands there is no closed form analytical solution. However, at each time step of the DE solver the $V_{\mathrm{spt}}$ value does not change significantly $(<0.1 \mathrm{ml})$ from the previous value. Thus, given the previous $V_{\mathrm{spt}}$ value, denoted $V_{\mathrm{spt}, \mathrm{old}}$, the exponential terms $e^{\lambda_{\mathrm{spt}} V_{\mathrm{spt}}}, e^{\lambda_{\mathrm{lvf}} V_{\mathrm{spt}}}$ and $e^{-\lambda_{\mathrm{rvf}} V_{\mathrm{spt}}}$ can be 
approximated by the Equations:

$$
\begin{aligned}
e^{\lambda_{\mathrm{spt}} V_{\mathrm{spt}}} & =a_{\mathrm{spt}} V_{\mathrm{spt}}+b_{\mathrm{spt}} \\
e^{-\lambda_{\mathrm{lvf}} V_{\mathrm{spt}}} & =a_{\mathrm{lvf}} V_{\mathrm{spt}}+b_{\mathrm{lvf}} \\
e^{\lambda_{\mathrm{rvf}} V_{\mathrm{spt}}} & =a_{\mathrm{rvf}} V_{\mathrm{spt}}+b_{\mathrm{rvf}}
\end{aligned}
$$

where $a_{\mathrm{spt}}, b_{\mathrm{spt}}, a_{\mathrm{lvf}}, b_{\mathrm{lvf}}, a_{\mathrm{rvf}}, b_{\mathrm{rvf}}$ are each a function of $V_{\mathrm{spt}, \mathrm{old}}$ and defined:

$$
\begin{aligned}
x_{1} & =V_{\mathrm{spt}, \mathrm{old}}+\Delta V_{\mathrm{spt}}, \quad x_{2}=V_{\mathrm{spt}, \mathrm{old}}-\Delta V_{\mathrm{spt}} \\
a_{\mathrm{spt}} & =\frac{e^{\lambda_{\mathrm{spt}} x_{2}}-e^{\lambda_{\mathrm{spt}} x_{1}}}{x_{2}-x_{1}}, \quad b_{\mathrm{spt}}=e^{\lambda_{\mathrm{spt}} x_{1}}-\left(e^{\lambda_{\mathrm{spt}} x_{2}}-\frac{e^{\lambda_{\mathrm{spt}} x_{1}}}{x_{2}-x_{1}} x_{1}\right) \\
a_{\mathrm{lvf}} & =\frac{e^{\lambda_{\mathrm{lvf}} x_{2}}-e^{\lambda_{\mathrm{lvf}} x_{1}}}{x_{2}-x_{1}}, \quad b_{\mathrm{lvf}}=e^{\lambda_{\mathrm{lvf}} x_{1}}-\left(e^{\lambda_{\mathrm{lvf}} x_{2}}-\frac{e^{\lambda_{\mathrm{lvf}} x_{1}}}{x_{2}-x_{1}} x_{1}\right) \\
a_{\mathrm{rvf}} & =\frac{e^{\lambda_{\mathrm{rvf}} x_{2}}-e^{\lambda_{\mathrm{rvf}} x_{1}}}{x_{2}-x_{1}}, \quad b_{\mathrm{rvf}}=e^{\lambda_{\mathrm{rvf}} x_{1}}-\left(e^{\lambda_{\mathrm{rvf}} x_{2}}-\frac{e^{\lambda_{\mathrm{rvf}} x_{1}}}{x_{2}-x_{1}} x_{1}\right)
\end{aligned}
$$

and $\Delta V_{\mathrm{spt}}$ is given the value of $0.1 \mathrm{ml}$.

Equations (58)-(59) are derived from finding the equation of the straight line joining the two points $\left(x_{2}, e^{\lambda_{\mathrm{spt}} x_{2}}\right)$ to $\left(x_{1}, e^{\lambda_{\mathrm{spt}} x_{1}}\right)$. Equations (60) and (61) are derived in a similar way. Equation (54) can thus be rewritten:

$$
\begin{aligned}
& e(t) E_{\mathrm{es}, \mathrm{spt}}\left(V_{\mathrm{spt}}-V_{\mathrm{d}, \mathrm{spt}}\right)+(1-e(t)) P_{0, \mathrm{spt}}\left(\left(a_{\mathrm{spt}} V_{\mathrm{spt}}+b_{\mathrm{spt}}\right) e^{-\lambda_{\mathrm{spt}} V_{0, \mathrm{spt}}}-1\right) \\
& =e(t) E_{\mathrm{es}, \mathrm{lvf}}\left(V_{\mathrm{lv}}-V_{\mathrm{spt}}\right)+(1-e(t)) P_{0, \mathrm{lvf}}\left(\left(a_{\mathrm{lvf}} V_{\mathrm{spt}}+b_{\mathrm{lvf}}\right) e^{\lambda_{\mathrm{lvf}} V_{\mathrm{lv}}}-1\right) \\
& -e(t) E_{\mathrm{es}, \mathrm{rvf}}\left(V_{\mathrm{rv}}+V_{\mathrm{spt}}\right)-(1-e(t)) P_{0, \mathrm{rvf}}\left(\left(a_{\mathrm{rvf}} V_{\mathrm{spt}}+b_{\mathrm{lvf}}\right) e^{\lambda_{\mathrm{rvf}} V_{\mathrm{rv}}}-1\right)
\end{aligned}
$$


Equation (62) is linear in $V_{\text {spt }}$ and thus has a unique closed form solution defined:

$$
\begin{aligned}
& V_{\mathrm{spt}}=\left\{( 1 - e ( t ) ) \left(P_{0, \mathrm{spt}}\left(e^{-\lambda_{\mathrm{spt}} V_{0, \mathrm{spt}}} b_{\mathrm{spt}}-1\right)-P_{0, \mathrm{lvf}}\left(e^{\lambda_{\mathrm{lvf}} V_{\mathrm{lv}}} b_{\mathrm{lvf}}-1\right)\right.\right. \\
& \left.\left.+P_{0, \mathrm{rvf}}\left(e^{\lambda_{\mathrm{rvf}} V_{\mathrm{rv}}} b_{\mathrm{rvf}}-1\right)\right)-e(t)\left(E_{\mathrm{es}, \mathrm{spt}} V_{\mathrm{d}, \mathrm{spt}}+E_{\mathrm{es}, \mathrm{lvf}} V_{\mathrm{lv}}-E_{\mathrm{es}, \mathrm{rvf}} V_{\mathrm{rv}}\right)\right\} / \\
& \left\{(1-e(t))\left(-P_{0, \mathrm{spt}} e^{-\lambda_{\mathrm{spt}} V_{0, \mathrm{spt}}} a_{\mathrm{spt}}+P_{0, \mathrm{lvf}} e^{\lambda_{\mathrm{lvf}} V_{\mathrm{lv}}} a_{\mathrm{lvf}}-P_{0, \mathrm{vrvf}} e^{\lambda_{\mathrm{rvf}} V_{\mathrm{rv}}} a_{\mathrm{rvf}}\right)\right. \\
& \left.\quad-e(t)\left(E_{\mathrm{es}, \mathrm{spt}}+E_{\mathrm{es}, \mathrm{lvf}}+E_{\mathrm{es}, \mathrm{rvf}}\right)\right\}
\end{aligned}
$$

Thus, the requirement to solve the non-linear Equation (54) numerically at each time step is avoided, significantly reducing the computation required to simulate the full CVS model with septum interaction.

\section{Results}

A healthy human is simulated using the Heaviside function formulation defined by Equations (40)-(43) and then using the event solver method as described in [11, 12]. Figure 4 shows the volume in the left ventricle over one heart beat for both methods. They are essentially overlaid and the total mean percentage difference in the two model's continuous outputs is $0.046 \%$ with a standard deviation of $0.104 \%$. The full model's pressure and volume outputs are shown in Table 1. These results agree well with values reported in medical physiology texts e.g. [16]. They also match results from the original solution method of [11-13]. As shown in Table 2, the initial Heaviside formulation is 1.5 times faster than the previous event solver method, requiring 70 seconds for a 20-beat simulation instead of 102 seconds.

Next the simpler Heaviside formulation defined by Equations (50)-(53) is simulated for the healthy human. The method is again run for 20 heart beats. Figure 5 shows the flows for the left ventricle in the filling stage $\left(Q_{\mathrm{mt}}>0\right)$ and ejection stage $\left(Q_{\mathrm{av}}>0\right)$ for both of the Heaviside methods over the $20^{\text {th }}$ heart beat. A noticeable 
error in the simpler Heaviside method can be seen to occur at the beginning of each ejection cycle at $t \sim 0.3$. This error is due to a transient dynamic resulting from a non-zero initial condition. This error can be significantly reduced by running the simpler Heaviside formulation for 19 heart beats then running the model one more heart beat using the first Heaviside method defined by Equations (40)-(43). The result is shown in Figure 6 where the curves are overlaid with no error. Table 2 shows that the computationally simpler Heaviside method is approximately 4 times faster than the previous event solver method of [11-13] and 2.6 times faster than the initial Heaviside formulation. Hence, reducing the computational evaluation of Heaviside functions at each time step by $67 \%$ provided a significant improvement.

The analytical formula for calculating $V_{\text {spt }}$, defined by Equation (63), is now tested. Table 2 shows that combining both methods gives a 24 times speed increase over the previous method, and a $6 \times$ improvement on the simpler Heaviside method alone. Note, in all cases presented until now the analytical solution for $V_{\mathrm{spt}}$ has not been employed. Hence, the impact of this added solution method is dramatic, with a 20-beat simulation requiring only 4.3 , of the original approaches 101.9 , seconds to complete.

The accuracy of the simpler Heaviside method plus analytical solution for $V_{\mathrm{spt}}$ is performed for a healthy human and four disease states: mitral and aortic stenosis, pulmonary embolism and septic shock. Comparisons are then made with the first Heaviside method presented. Mitral and aortic stenosis are simulated by multiplying the mitral and aortic resistances by 2 and 5 , respectively. Pulmonary embolism and septic shock are simulated by multiplying the pulmonary and systemic resistances by 2 and 0.5 , respectively. Each simulation has been shown to accurately capture the reported physiological trends $[11-13,17]$. Table 3 shows the total mean percentage difference error in all the model's outputs. The mean errors are no greater than $0.2 \%$ showing the method is very accurate and that the improved computational approach does not impact model validity or accuracy. 


\section{Discussion and Conclusions}

The method presented efficiently represents the valve law using Heaviside functions, includes an analytical formula for ventricular interaction and is 24 times faster than the previous method that uses a computationally costly, zero-finding event solver in $[11,12]$. The new method is tested on a model of a healthy human and four disease states: mitral and aortic stenosis, pulmonary embolism and septic shock. Results show that the model is very accurate and is within a total mean error of $0.2 \%$ of the previous method, well within any potential measurement error.

The non-linear equation for ventricular interaction required computationally expensive non-linear numerical function solver routines at every time step of the DE. This step is transformed into a linear equation for $V_{\text {spt }}$ with a closed form analytical solution. This change alone is responsible for approximately a $6 \times$ speed increase compared to a $4 \times$ speed increase for the simpler Heaviside formulation, totalling $24 \times$ improvement over the previous method when combined. The approach is computationally very fast and accurate, which is critical in the process of simulating a large number of therapy treatment combinations to find the best treatment. Clinically, medical staff can then obtain rapid feedback on a standard desktop computer and longer, useful simulations can be readily performed in $1-2$ minutes, making clinical application of the model more realistic.

\section{References}

[1] C. S. Peskin and D. M. McQueen. Cardiac fluid dynamics. Crit Rev Biomed Eng, 20(5-6):451-9, 1992.

[2] D. M. McQueen, C. S. Peskin, and E. L. Yellin. Fluid dynamics of the mitral valve: physiological aspects of a mathematical model. Am J Physiol, 242(6):H1095-110, 1982. 
[3] I.J. Legrice, P.J. Hunter, and B.H. Smaill. Laminar structure of the heart: a mathematical model. Am J Physiol, 272:H2466-76, 1997.

[4] B. Smaill and P. Hunter. Structure and function of the diastolic heart: Material properties of passive myocardium. In Leon Glass, Peter Hunter, and Andrew McCulloch, editors, Theory of Heart, pages 1-29. Springer-Verlag, Harrisonburg, 1991.

[5] J. Amoore, W. Santamore, W. Corin, and D. George. Computer simulation of the effects of ventricular interdependence on indices of left ventricular systolic function. J. Biomed. Eng, 14:257-262, 1992.

[6] R. Beyar, M.J. Hausknecht, H.R. Halperin, F.C. Yin, and M.L. Weisfeldt. Interaction between cardiac chambers and thoracic pressure in intact circulation. Am. J. Physiol, 22(253):H1240-52, 1987.

[7] D. Chung, S. Niranjan, J. Clark, A. Bidani, and W. Johnston. A dynamic model of ventricular interaction and pericardial influence. J. Physiol., 14(272):H2924H2962, 1997.

[8] M. Ursino. A mathematical model of the carotid baroregulation in pulsating conditions. IEEE Trans Biomed Eng, 46(4):382-92, 1999.

[9] N. Stergiopulos, P. Segers, and N. Westerhof. Use of pulse pressure method for estimating total arterial compliance in vivo. Am. J. Physiol., $276(2 \mathrm{Pt}$ 2):H424-8, 1999.

[10] Daniel Burkhoff and John V. Tyberg. Why does pulmonary venous pressure rise after onset of lv dysfunction: a theoretical analysis. Am. J. Physiol., 265:H1819H1828, 1993.

[11] B.W. Smith. Minimal Haemodynamic Modelling of the Heart and Circulation for Clinical Application. PhD thesis, University of Canterbury, 2004. 
[12] B.W. Smith, J.G. Chase, R. I. Nokes, G.M. Shaw, and G. Wake. Minimal haemodynamic system model including ventricular interaction and valve dynamics. Med. Eng. Phys, 26(2):131-139, 2004.

[13] B.W. Smith, J.G. Chase, G.M. Shaw, and R.I. Nokes. Experimentally verified minimal cardiovascular system model for rapid diagnostic assistance. Control Engineering Practice, in press, 2005.

[14] C.E. Hann, J.G. Chase, G.M. Shaw, and B.W. Smith. Identification of patient specific parameters for a minimal cardiac model.

Proc 26 th International Conf of IEEE Engineering in Med and Biology Society (EMBS 2004), San Francisco, CA, Sept 1-5, pages 813-816, 2004.

[15] C.E. Hann, J.G. Chase, J. Lin, T. Lotz, C.V. Doran, and G.M. Shaw. Integralbased parameter identification for long-term dynamic verification of a glucoseinsulin system model. Computer Methods and Programs in Biomedicine, $77(3): 259-270,2005$.

[16] A.C. Guyton and J.E. Hall. Textbook of Medical Physiology. Philadelphia: W.B. Saunders Company, 2001.

[17] E. Braunwald. Heart Disease, A text book of cardiovascular medicine, 5th edition. W.B. Saunders Company, Philadelphia, 1997. 


\begin{tabular}{|l|l|}
\hline Description & Output \\
\hline Volume in left ventricle & $111.7 / 45.7 \mathrm{ml}$ \\
Volume in right ventricle & $112.2 / 46.1 \mathrm{ml}$ \\
Max $P_{\mathrm{lv}}$ & $119.2 \mathrm{mmHg}$ \\
Max $P_{\mathrm{rv}}$ & $26.2 \mathrm{mmHg}$ \\
Pressure in aorta & $116.6 / 79.1 \mathrm{mmHg}$ \\
Pressure in pulmonary artery & $25.7 / 7.8 \mathrm{mmHg}$ \\
Average pressure in pulmonary vein & $2.0 \mathrm{mmHg}$ \\
Average pressure in vena cava & $2.0 \mathrm{mmHg}$ \\
\hline
\end{tabular}

Table 1: Healthy human model outputs using the Heaviside model formulation of Equations (40)-(43).

\begin{tabular}{|l|l|l|}
\hline Method & CPU time $(s)$ & Speed increase $(\times)$ \\
\hline Event solver & 101.9 & \\
First Heaviside & 70.0 & 1.5 \\
Simpler Heaviside & 26.3 & 3.9 \\
Simpler Heaviside + & 4.3 & 23.7 \\
analytical $V_{\text {spt }}$ formula & & \\
\hline
\end{tabular}

Table 2: Computational speeds for simulating full heart model (20 beats) with the different Heaviside model formulations presented and comparison to solutions using the methods of [11-13].

\begin{tabular}{|l|l|l|}
\hline Disease state & $\begin{array}{l}\text { Total percentage error } \\
\text { Mean }\end{array}$ & Standard deviation \\
\hline Healthy & 0.14 & 0.21 \\
Mitral Stenosis & 0.20 & 0.28 \\
Aortic Stenosis & 0.15 & 0.22 \\
Pulmonary Embolism & 0.18 & 0.31 \\
Septic Shock & 0.09 & 0.15 \\
\hline
\end{tabular}

Table 3: Comparing the accuracy of the simpler Heaviside method plus analytical $V_{\text {spt }}$ formula to the first Heaviside method, using Equations (50)-(53) and Equation (63). 


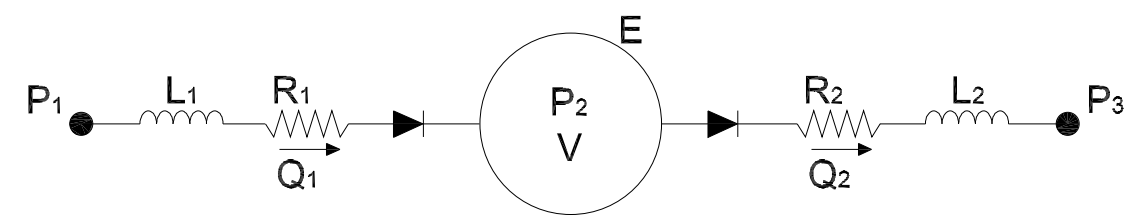

Figure 1: The single cardiac chamber model

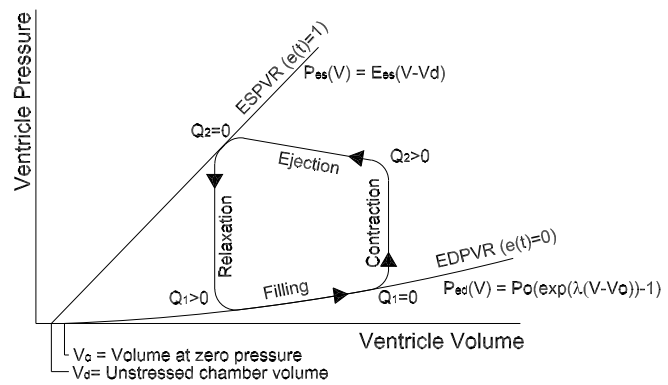

(a)

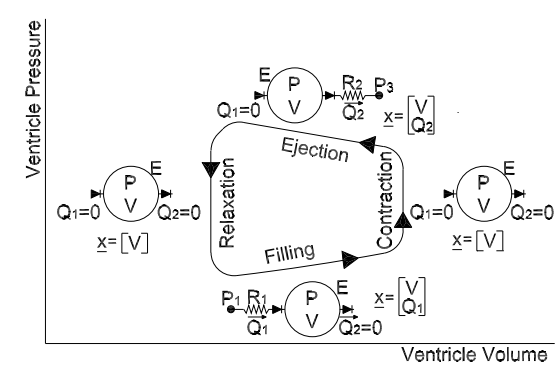

(b)

Figure 2: An example of a pressure-volume diagram with the different states of the single cardiac chamber model included. Right side shows model state at each part of the cardiac cycle.

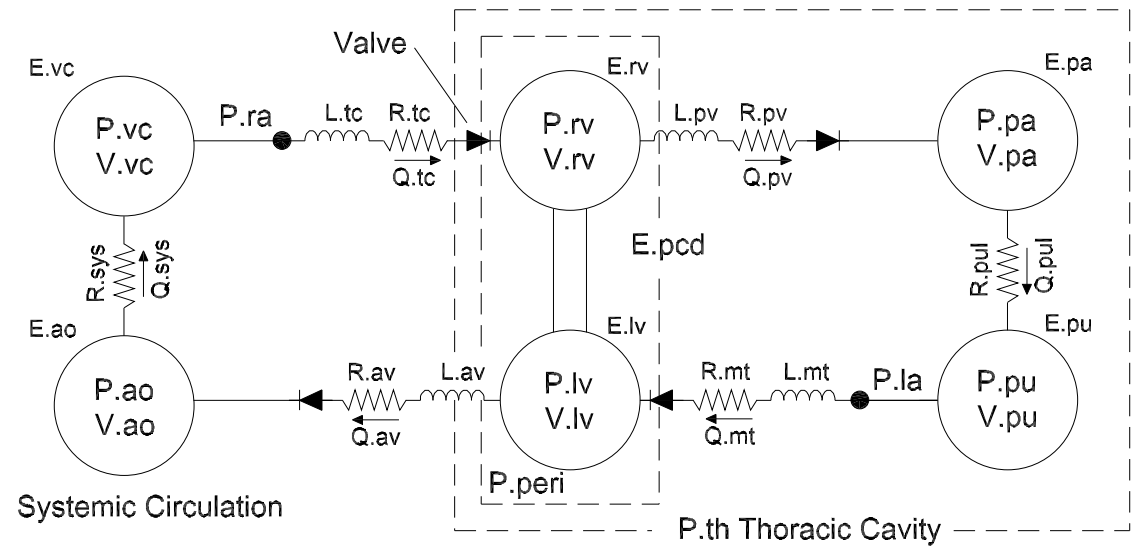

Figure 3: The presented closed loop model of the cardiovascular system. 


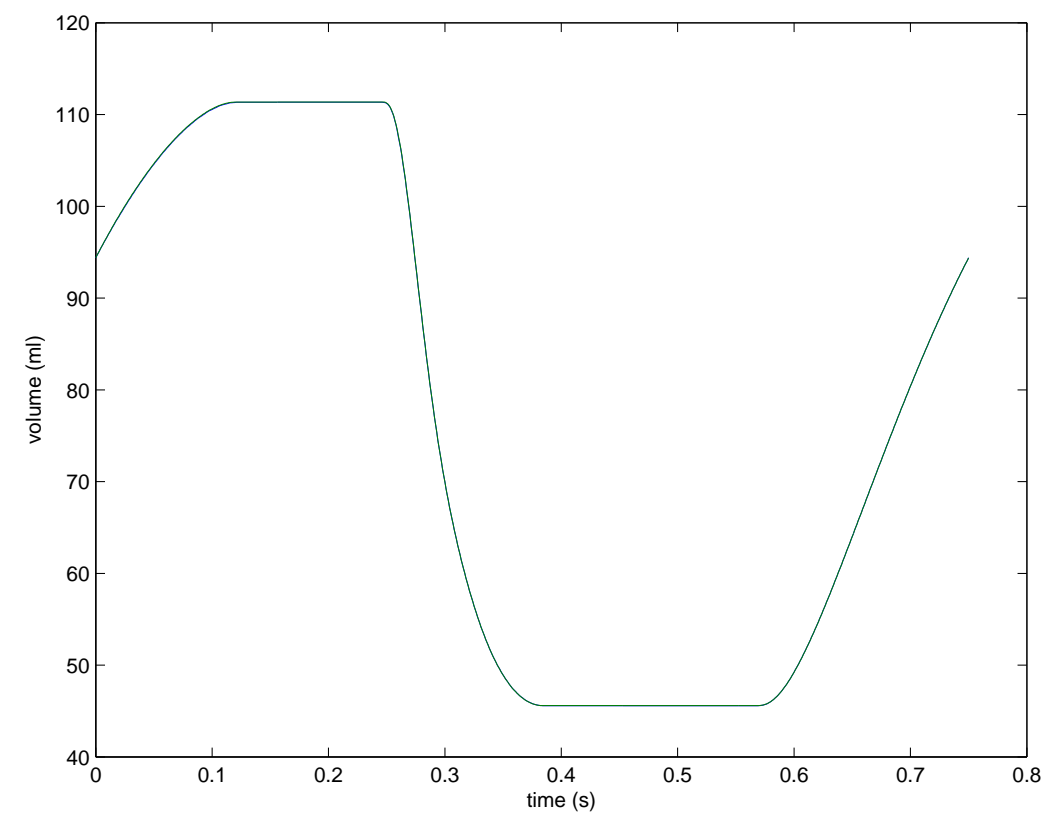

Figure 4: Volume in the left ventricle for the Heaviside method of Equations (6)-(23) and event solver method of $[11,12]$. The curves are effectively identical. 


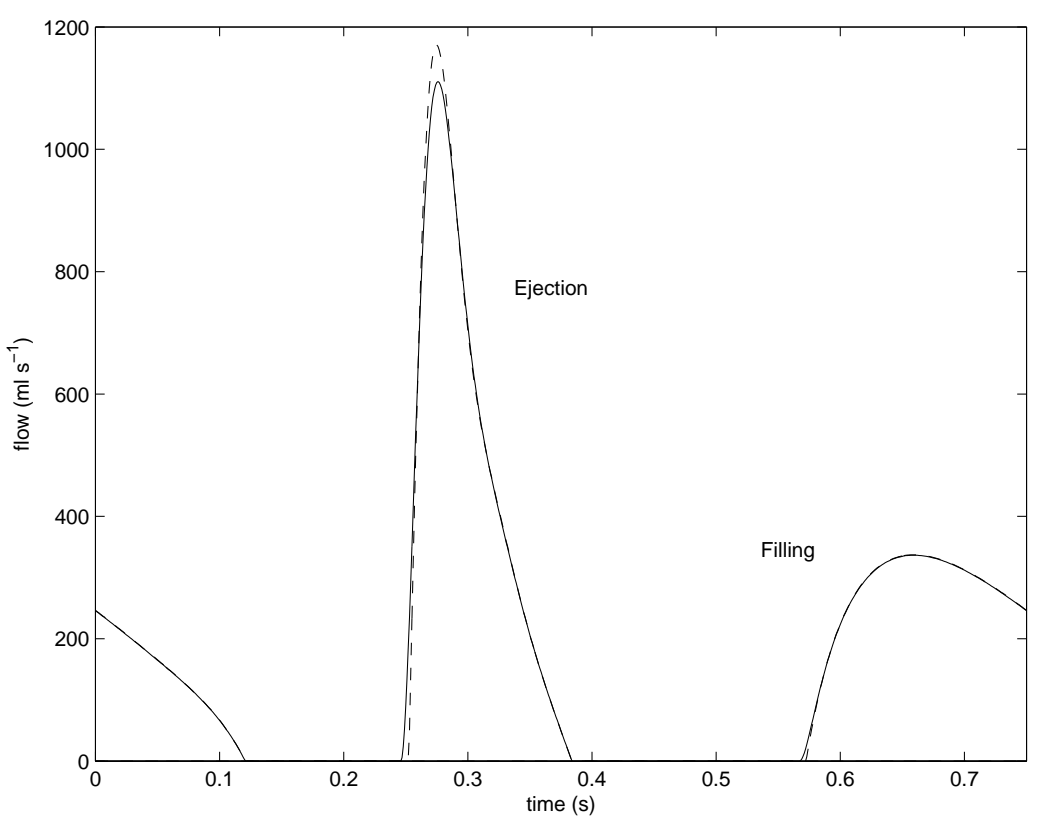

Figure 5: The flow through the mitral valve $\left(Q_{\mathrm{mt}}\right)$ and aortic valve $\left(Q_{\mathrm{av}}\right)$ for the first Heaviside method (solid line) and the simpler Heaviside method of Equations (50)(53) (dashed line) for the $20^{\text {th }}$ heart beat. The simpler Heaviside method employs the analytical expression for $V_{\text {spt }}$ in Equation (63) where the first Heaviside method does not. 


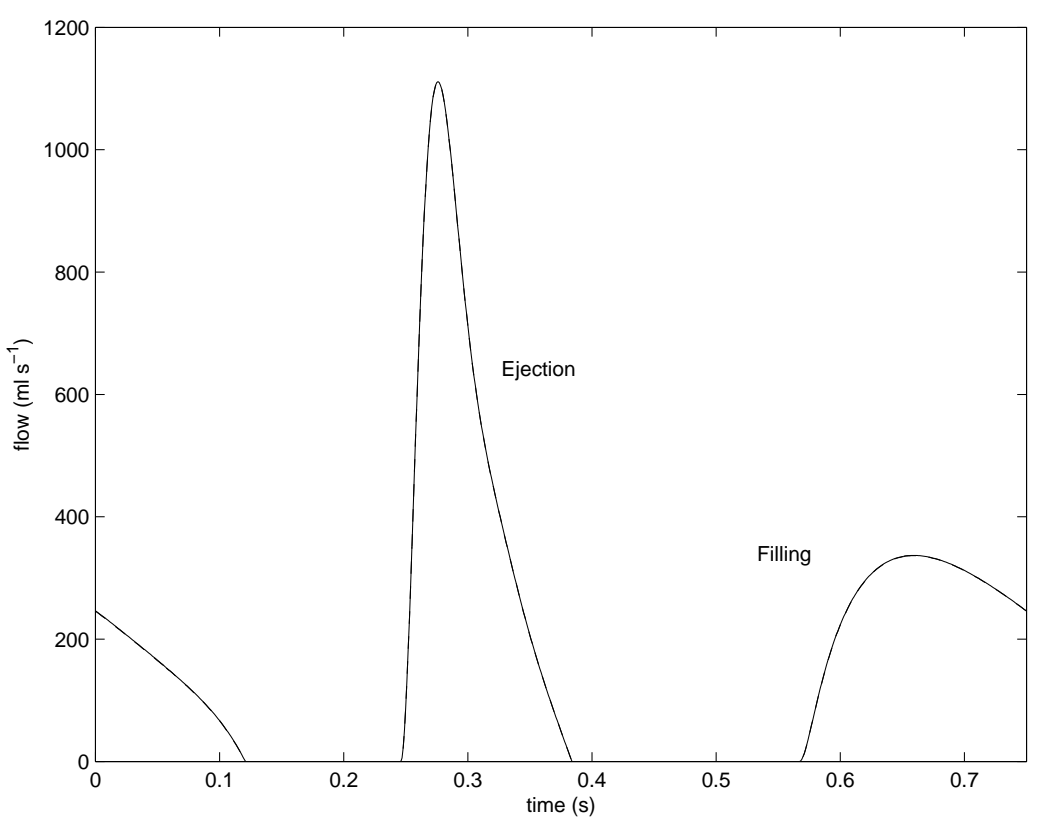

Figure 6: The flow through the mitral valve $\left(Q_{\mathrm{mt}}\right)$ and aortic valve $\left(Q_{\mathrm{av}}\right)$ after running the simpler Heaviside method of Equations (50)-(53) for 19 heart beats followed by the first Heaviside method of Equations (8)-(19) for one heart beat (dashed line). The first Heaviside method, run over 20 heart beats is the solid line, and the results are effectively identical. The simpler Heaviside method (19 beats) + first Heaviside method (1 beat) employs the analytical expression for $V_{\text {spt }}$ in Equation (63) where the first Heaviside method run over the 20 heart beats does not. 